\title{
Anti-inflammatory Effects of the Octapeptide NAP in Human Microbiota-Associated Mice Suffering from Subacute Ileitis
}

\author{
Ulrike Escher $^{1}$, Eliezer Giladi ${ }^{2}$, Ildikò R. Dunay ${ }^{3}$, Stefan Bereswill ${ }^{1}$, \\ Illana Gozes ${ }^{2 \dagger}$ and Markus M. Heimesaat ${ }^{1{ }^{\dagger *}}$ \\ ${ }^{1}$ Charité - Universitätsmedizin Berlin, corporate member of Freie Universität Berlin, Humboldt-Universität zu Berlin, \\ and Berlin Institute of Health, Institute of Microbiology and Infection Immunology, Berlin, Germany \\ ${ }^{2}$ Department of Human Molecular Genetics and Biochemistry, Sackler School of Medicine, Tel Aviv University, Tel Aviv, Israel \\ ${ }^{3}$ Institute of Inflammation and Neurodegeneration, Medical Faculty, Otto-von-Guericke University Magdeburg, Magdeburg, Germany
}

Received: 17 March 2018; accepted: 11 April 2018

\begin{abstract}
The octapeptide NAP is well known for its neuroprotective properties. We here investigated whether NAP treatment could alleviate pro-inflammatory immune responses during experimental subacute ileitis. To address this, mice with a human gut microbiota were perorally infected with one cyst of Toxoplasma gondii (day 0) and subjected to intraperitoneal synthetic NAP treatment from day 1 until day 8 postinfection (p.i.). Whereas placebo (PLC) control animals displayed subacute ileitis at day 9 p.i., NAP-treated mice exhibited less pronounced pro-inflammatory immune responses as indicated by lower numbers of intestinal mucosal $\mathrm{T}$ and $\mathrm{B}$ lymphocytes and lower interferon (IFN)- $\gamma$ concentrations in mesenteric lymph nodes. The NAP-induced anti-inflammatory effects were not restricted to the intestinal tract but could also be observed in extra-intestinal including systemic compartments, given that pro-inflammatory cytokines were lower in liver, kidney, and lung following NAP as compared to PLC application, whereas at day 9 p.i., colonic and serum interleukin (IL)-10 concentrations were higher in the former as compared to the latter. Remarkably, probiotic commensal bifidobacterial loads were higher in the ileal lumen of NAP as compared to PLC-treated mice with ileitis. Our findings thus further support that NAP might be regarded as future treatment option directed against intestinal inflammation.

Keywords: octapeptide NAP, activity-dependent neuroprotective protein (ADNP), host-pathogen interactions, Toxoplasma gondii, subacute ileitis, host immunity, human gut microbiota, fecal microbiota transplantation, secondary abiotic (gnotobiotic) mice, intestinal, extra-intestinal and systemic immune responses
\end{abstract}

\section{Introduction}

The octapeptide NAP consists of eight amino acids (namely, NAPVSIPQ) and was initially identified as a biologically active fragment of the activity-dependent neuroprotective protein (ADNP) [1]. Human ADNP is not only expressed within the central nervous system (CNS), but also in the spleen, in peripheral blood leukocytes, and in macrophages [2-4]. The neuroprotective effects of NAP have been shown in vitro and in several in vivo models of neuronal morbidities including Alzheimer's disease, stroke, closed head injury, fetal alcohol syndrome, and neonatal hypoxia [1, 5-9], as potential underlying mechanisms for the beneficial properties exerted by NAP immunomodulatory and anti-oxidative effects have been proposed [10]. Regardless, information regarding anti-inflammatory effects of NAP beyond the CNS is scarce.

In our previous mouse studies applying acute intestinal inflammation models within different anatomic compartments and with a fatal outcome within 1 week post induction, we were able to show that NAP application resulted in potent anti-inflammatory effects in both, acute small intestinal [11] and acute large intestinal inflammation [12]. Remarkably, the anti-inflammatory effects of NAP treatment were not restricted to the intestinal tract, given that extra-intestinal and even systemic collateral damages of inflammation could be alleviated [11, 12]. This prompted us to elucidate potential immune-modulatory actions of NAP in another gut inflammation model but of less acute severity. We therefore applied the very recently established non-lethal subacute ileitis

\footnotetext{
* Corresponding author: Markus M. Heimesaat; markus.heimesaat@charite.de

Shared senior authorship.
}

mouse model [13]. Within 9 days following peroral low-dose Toxoplasma gondii infection, susceptible mice harboring a human commensal gut microbiota develop subacute $\mathrm{T}$ cell driven ileitis that is characterized by increased gut epithelial apoptosis and pro-inflammatory cytokine secretion in intestinal and extra-intestinal compartments, whereas anti-inflammatory interleukin (IL)-10 cytokine expression and accelerated regenerative epithelial cell responses constitute counter-regulatory measures in order to limit immunopathological sequelae [13]. In the present study, we show that intraperitoneal application of synthetic NAP alleviated intestinal as well as extra-intestinal pro-inflammatory immune responses upon low-dose $T$. gondii infection, whereas intestinal and systemic anti-inflammatory IL-10 secretion was reinforced to counteract ileitis.

\section{Materials and Methods}

Generation of Human Microbiota-associated Mice. Female C57BL/6j wildtype mice were bred and housed in the facilities of the "Forschungseinrichtungen für Experimentelle Medizin" (FEM, Charité - Universitätsmedizin, Berlin, Germany) under specific pathogen-free (SPF) conditions.

With respect to their microbiota composition, "humanized" mice were generated as previously described [14-16]. In brief, the gut microbiota of female 8-week-old mice was virtually depleted following a 6-week course of quintuple broad-spectrum antibiotic treatment (ad libitum via autoclaved drinking water) [17]. Three days after cessation of the antibiotic cocktail (to assure antimicrobial washout), thus generated secondary abiotic mice were associated with human gut microbiota by peroral fecal

This is an open-access article distributed under the terms of the Creative Commons Attribution-NonCommercial 4.0 International License (https://creativecommons.org/licenses/by-nc/4.0/), which permits unrestricted use, distribution, and reproduction in any medium for non-commercial purposes, provided the original author and source are credited, a link to the CC License is provided, and changes - if any - are indicated. 
microbiota transplantation (FMT) as described earlier [14-16]. To assure human commensal bacterial establishment within the murine intestinal ecosystem, mice were kept for 2 weeks until ileitis induction.

Induction of Subacute Ileitis and Treatment of Mice. On day 0 , subacute ileitis was induced by peroral low-dose T. gondii ME49 strain infection of mice via gavage of only one cyst in $0.3 \mathrm{~mL}$ brain suspension as reported earlier [13]. From day 1 until day 8 postinfection (p.i.), mice were either treated intraperitoneally with synthetic NAP $(1.0 \mathrm{mg}$ per $\mathrm{kg}$ body weight per day) or placebo (PLC; sterile phosphatebuffered saline [PBS], Gibco, Life Technologies, UK) once daily. A potential antimicrobial effect of the applied NAP solution was excluded as reported earlier [11].

Sampling Procedures. Mice were sacrificed by isofluran treatment (Abbott, Greifswald, Germany) at day (d) 9 p.i. Cardiac blood, ileal luminal samples, and ex vivo biopsies derived from liver, kidney, lung, mesenteric lymph nodes (MLNs), ileum, and colon were taken under sterile conditions and collected from each mouse in parallel for microbiological, immunological, and immunohistochemical analyses.

Immunohistochemistry. Ex vivo biopsies derived from the terminal ileum and colon were immediately fixed in 5\% formalin and embedded in paraffin. In situ immunohistochemical analyses of $5 \mu \mathrm{m}$ thin paraffin sections from small and large intestinal ex vivo biopsies were performed as stated elsewhere [12, 18-20]. Primary antibodies against CD3 (no. IR50361-2, Dako, Santa Clara, CA, USA; 1:5) and B220 (no. 14-0452-81, eBioscience; 1:200) were used. For each animal, the average numbers of positively stained cells within at least six high power fields (HPF, $0.287 \mathrm{~mm}^{2}, 400 \times$ magnification) were determined microscopically by an independent investigator.

Cytokine Measurements. Ex vivo biopsies derived from MLN, liver (approximately $1 \mathrm{~cm}^{2}$ ), kidney (one half after longitudinal cut), and lung were placed in 24-well flat-bottom culture plates (Nunc, Wiesbaden, Germany) containing $500 \mu \mathrm{L}$ serum-free RPMI 1640 medium (Gibco, Life Technologies) supplemented with penicillin $(100 \mathrm{U} / \mathrm{mL})$ and streptomycin (100 $\mu \mathrm{g} / \mathrm{mL}$; PAA Laboratories). After $18 \mathrm{~h}$ at $37^{\circ} \mathrm{C}$, culture supernatants and serum samples were tested for interferon (IFN)- $\gamma$, monocyte chemoattractant protein (MCP)-1, IL-6, IL-12p70, and IL-10 by the mouse inflammation cytometric bead assay (CBA; BD Biosciences, Heidelberg, Germany) on a BD FACSCanto II flow cytometer (BD Biosciences) as stated elsewhere [11, 14, 21].

Cultural Survey of Bacterial Translocation to Extraintestinal Compartments. For comprehensive quantitative and qualitative survey of viable bacteria translocating from the intestinal tract to extra-intestinal compartments including MLN, liver, kidney, and lung, respective intestinal samples and ex vivo biopsies were homogenized in sterile PBS and analyzed in serial dilutions on respective solid media as previously described [17, $18,22]$. In addition, cardiac blood was directly streaked onto solid media. Bacteria were grown at $37{ }^{\circ} \mathrm{C}$ for at least 2 days under aerobic, microaerobic, and anaerobic conditions.

Molecular Analysis of Gut Microbiota. Fresh ileal luminal samples were immediately snap-frozen in liquid nitrogen and stored at $-80{ }^{\circ} \mathrm{C}$ until further processing. DNA was extracted from intestinal luminal samples as reported earlier [17, 23]. In brief, DNA was quantified by using Quant-iT PicoGreen reagent (Invitrogen, UK) and adjusted to $1 \mathrm{ng} / \mu \mathrm{L}$. Then, the main bacterial groups abundant in the murine intestinal microbiota including enterobacteria, enterococci, lactobacilli, bifidobacteria, Bacteroides/Prevotella spp., Clostridium coccoides group, Clostridium leptum group, and total eubacterial loads were determined by quantitative real-time polymerase chain reaction (qRT-PCR) with species-, genera-, or group-specific 16S rRNA gene primers (Tib MolBiol, Germany) as reported previously [19, 24-27], and numbers of 16S rRNA gene copies per nanogram DNA of each sample were assessed.

Statistical Analysis. Medians, means, standard deviations, and significance levels using Mann-Whitney $U$ test were

\section{A T Lymphocytes (ILEUM)}

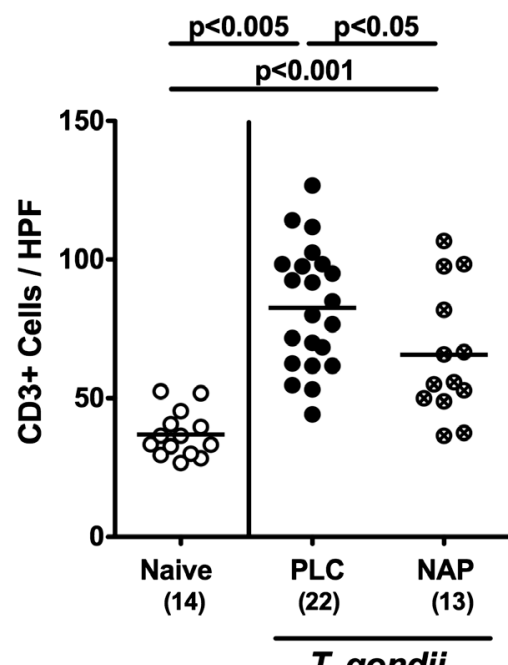

T. gondii
B T Lymphocytes
(COLON)

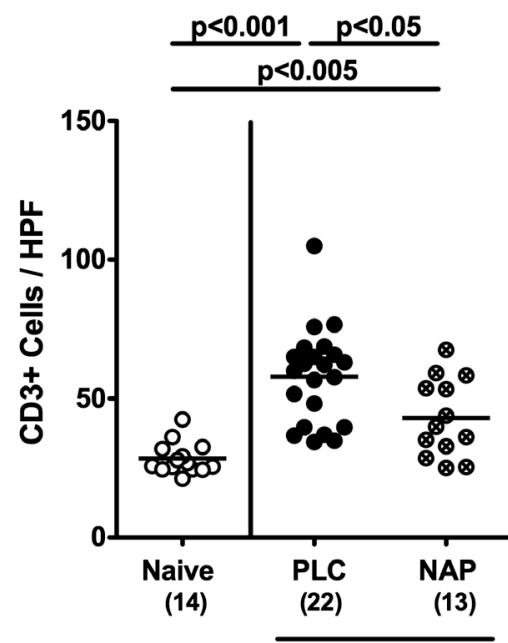

T. gondii

\section{B Lymphocytes (COLON)}

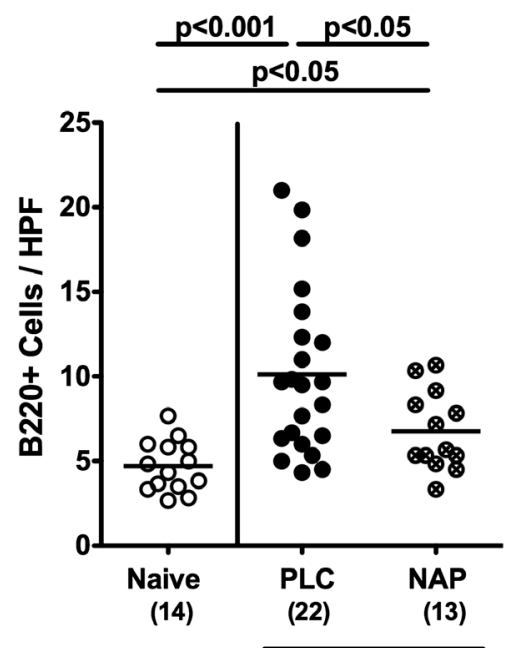

T. gondii

Figure 1. Intestinal immune cell responses upon NAP treatment of human microbiota-associated mice suffering from subacute ileitis. Subacute ileitis was induced in human microbiota-associated (hma) mice on day 0 by peroral low-dose T. gondii infection as described in Materials and Methods section. Hma mice were then treated with synthetic NAP (crossed circles) or placebo (PLC; black circles) from day 1 until day 8 postinfection (p.i.). At day 9 p.i., the average numbers of (A) ileal and (B) colonic T lymphocytes (positive for CD3) and of (C) colonic B lymphocytes (positive for B220) were determined microscopically in immunohistochemically stained intestinal paraffin sections from six high power fields (HPF, 400× magnification) per animal. Naive mice (i.e., without ileitis and without treatment; white circles) served as negative controls. Numbers of animals (in parentheses), means, and significance levels ( $p$ values) determined by Mann-Whitney $U$ test are indicated. Data shown were pooled from four independent experiments 


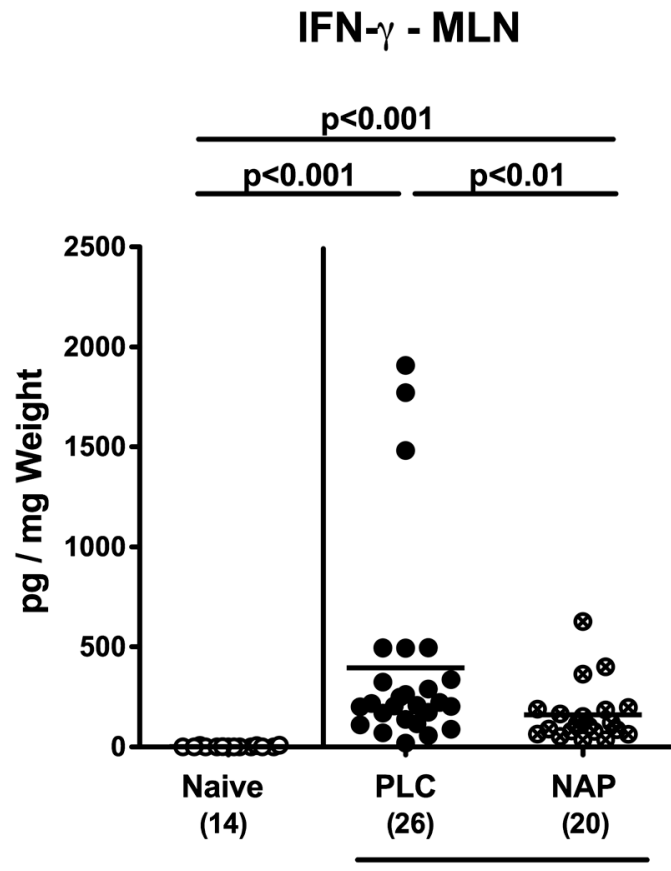

\section{T. gondii}

Figure 2. Intestinal pro-inflammatory cytokine response upon NAP treatment of human microbiota-associated mice suffering from subacute ileitis. Subacute ileitis was induced in human microbiota-associated (hma) mice on day 0 by peroral low-dose $T$. gondii infection as described in Materials and Methods section. Hma mice were then treated with synthetic NAP (crossed circles) or placebo (PLC; black circles) from day 1 until day 8 postinfection (p.i.). At day 9 p.i., secretion of the pro-inflammatory cytokine IFN- $\gamma$ was determined in ex vivo mesenteric lymph node (MLN) biopsies. Naive mice (i.e., without ileitis and without treatment; white circles) served as negative controls. Numbers of animals (in parentheses), means, and significance levels ( $p$ values) determined by Mann-Whitney $U$ test are indicated. Data shown were pooled from four independent experiments

A MCP-1 - LIVER

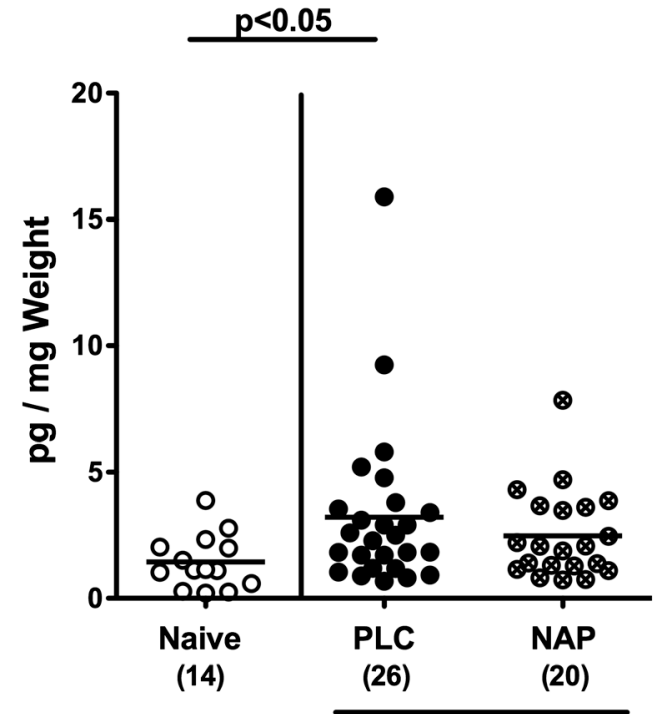

T. gondii determined using GraphPad Prism Software v6 (La Jolla, CA, USA). Two-sided probability $(p)$ values $\leq 0.05$ were considered significant. Experiments were repeated three times.

Ethics Statement. All animal experiments were conducted according to the European Guidelines for animal welfare (2010/63/EU) with approval from the commission for animal experiments headed by the "Landesamt für Gesundheit und Soziales“ (LaGeSo, Berlin, Germany, registration number G0145/10). Animal welfare was monitored twice daily by assessment of clinical conditions and weight loss of mice.

\section{Results}

NAP Treatment of Human Microbiota-associated Mice Suffering from Subacute Ileitis Results in less Pronounced Proinflammatory Immune Responses in the Intestinal Tract. In order to induce subacute ileitis, mice harboring a human commensal gut microbiota were perorally infected with a low dose of $T$. gondii on day 0 and subjected to intraperitoneal treatment with synthetic NAP or placebo from day 1 until day 8 p.i. At day 9 p.i., mice suffering from ileitis displayed increased numbers of $\mathrm{T}$ lymphocytes in their ileal as well as colonic mucosa and lamina propria $(p<0.005-0.001$; Figure 1A, B). These increases were, however, far less pronounced upon NAP treatment ( $p<0.05$ vs. placebo), which also held true for colonic B lymphocyte counts at day 9 p.i. ( $p<0.05$ vs. placebo; Figure 1C). The reduced intestinal immune cell responses following daily NAP challenge were accompanied by lower IFN- $\gamma$ concentrations in MLN of NAP as compared to placebotreated mice at day of necropsy ( $p<0.01$; Figure 2$)$.

NAP Treatment of Human Microbiota-associated Mice Suffering from Subacute Ileitis Results in Less Pronounced Pro-inflammatory Immune Responses in Extra-intestinal Compartments. We next addressed whether NAP could effectively exert anti-inflammatory properties beyond the intestinal tract. In fact, placebo-treated, but not NAP-treated, mice displayed increased secretion of pro-inflammatory cytokines

\section{B IL-6 - LIVER}

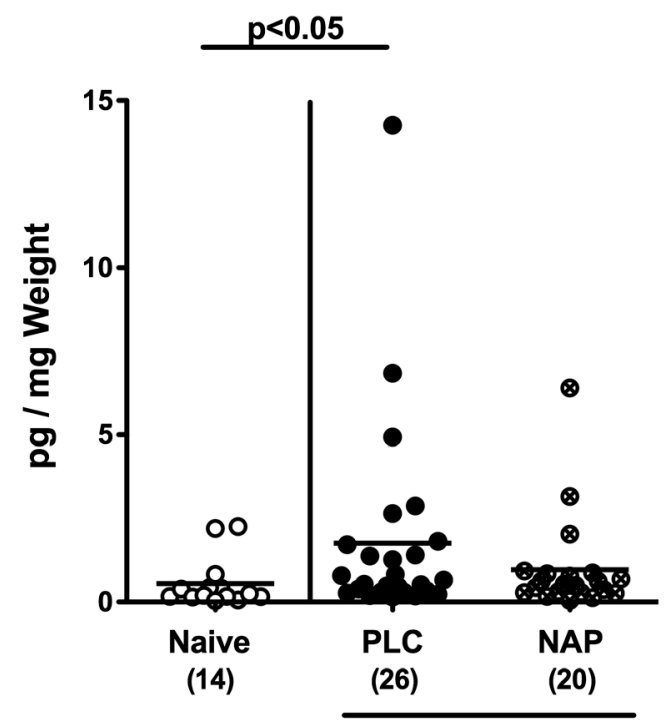

T. gondii

Figure 3. Hepatic pro-inflammatory cytokine responses upon NAP treatment of human microbiota-associated mice suffering from subacute ileitis. Subacute ileitis was induced in human microbiota-associated (hma) mice on day 0 by peroral low-dose $T$. gondii infection as described in Materials and Methods section. Hma mice were then treated with synthetic NAP (crossed circles) or placebo (PLC; black circles) from day 1 until day 8 postinfection (p.i.). At day 9 p.i., secretion of the pro-inflammatory cytokines (A) MCP-1 and (B) IL-6 was determined in ex vivo liver biopsies. Naive mice (i.e., without ileitis and without treatment; white circles) served as negative controls. Numbers of animals (in parentheses), means, and significance levels ( $p$ values) determined by Mann-Whitney $U$ test are indicated. Data shown were pooled from four independent experiments 
such as MCP-1 and IL-6 in liver ex vivo biopsies at day 9 p.i. $(p<0.05$; Figure 3), which was also true for IL-12p70 concentrations in kidneys ( $p<0.001$; Figure 4). Furthermore, mice suffering from subacute ileitis displayed multi-fold increased INF- $\gamma$ protein levels in their lungs $(p<0.001$; Figure 5), which were more than $50 \%$ lower upon NAP as compared to placebo treatment $(p<0.001$; Figure 5). Of note, we were not able to detect any viable bacteria that had translocated from the intestinal tract to extra-intestinal including systemic compartments.

NAP Treatment of Human Microbiota-associated Mice Suffering from Subacute Ileitis Results in More Distinct Intestinal as well as Systemic Anti-inflammatory Cytokine Secretion. To further underline the anti-inflammatory properties of NAP, we additionally assessed intestinal as well as systemic IL-10 secretion. As compared to naive control animals, NAPtreated, but not placebo-treated, human microbiota-associated (hma) mice with subacute ileitis displayed higher IL-10 concentrations in colonic ex vivo biopsies $(p<0.005$; Figure $6 \mathrm{~A}$ ) as well as in serum samples $(p<0.05$; Figure $6 \mathrm{~B})$ taken at day 9 p.i.

Hence, NAP exerts anti-inflammatory properties during subacute ileitis of (with respect to their gut microbiota) "humanized" mice that are not restricted to the intestinal tract but can also be observed in extra-intestinal including systemic compartments.

Bifidobacterial Loads are Higher Following NAP Treatment of Human Microbiota-associated Mice Suffering from Subacute Ileitis. We finally surveyed changes in the small intestinal microbiota composition during subacute ileitis development in NAP-treated hma mice applying quantitative culture-independent 16S rRNA based analyses. At day 9 p.i., mice of either cohort displayed higher loads of commensal enterobacteria (including Escherichia

\section{IL-12p70 - KIDNEY}

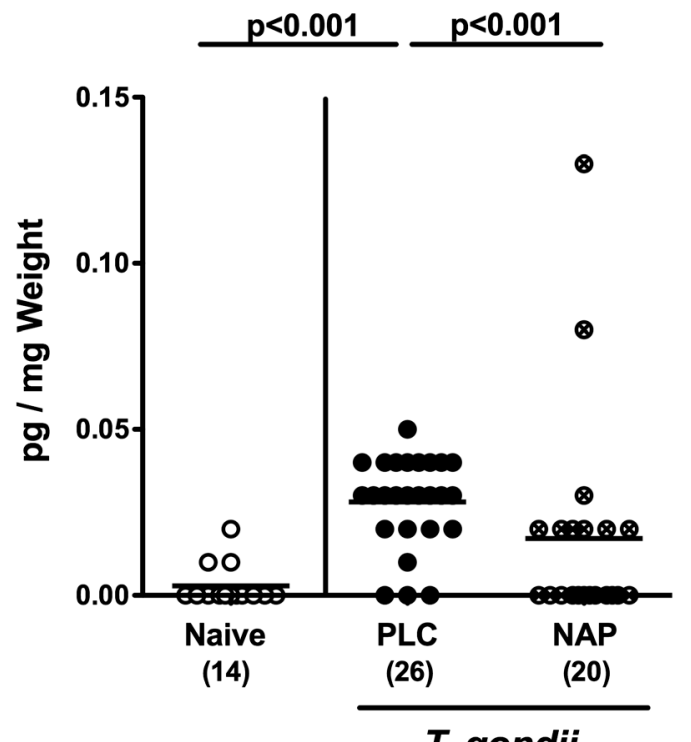

T. gondii

Figure 4. Renal pro-inflammatory cytokine response upon NAP treatment of human microbiota-associated mice suffering from subacute ileitis. Subacute ileitis was induced in human microbiota-associated (hma) mice on day 0 by peroral low-dose $T$. gondii infection as described in Materials and Methods section. Hma mice were then treated with synthetic NAP (crossed circles) or placebo (PLC; black circles) from day 1 until day 8 postinfection (p.i.). At day 9 p.i., secretion of the proinflammatory cytokine IL-12p70 was determined in ex vivo kidney biopsies. Naive mice (i.e., without ileitis and without treatment; white circles) served as negative controls. Numbers of animals (in parentheses), means, and significance levels ( $p$ values) determined by Mann-Whitney $U$ test are indicated. Data shown were pooled from four independent experiments
IFN- $\gamma$ - LUNG

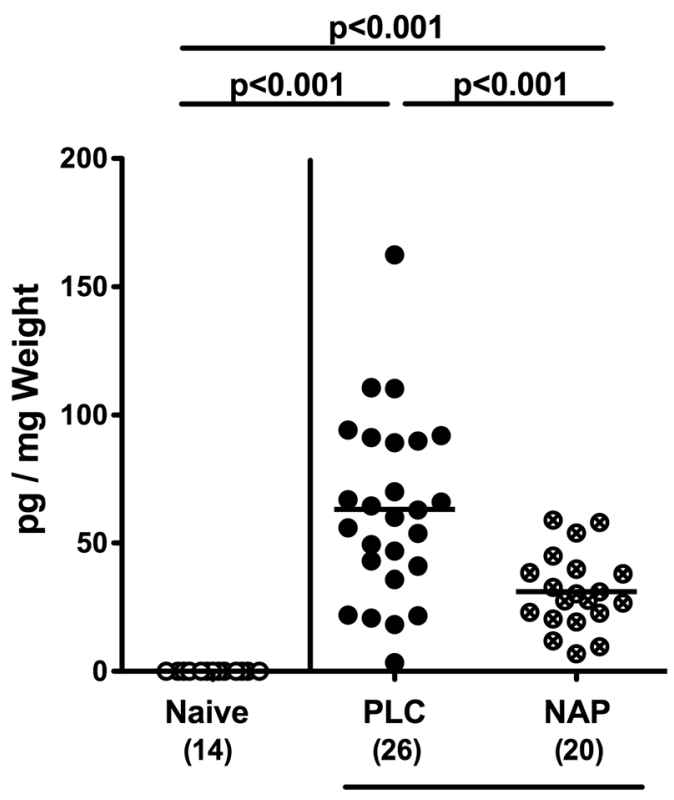

T. gondii

Figure 5. Pulmonary pro-inflammatory cytokine response upon NAP treatment of human microbiota-associated mice suffering from subacute ileitis. Subacute ileitis was induced in human microbiota-associated (hma) mice on day 0 by peroral low-dose T. gondii infection as described in Materials and Methods section. Hma mice were then treated with synthetic NAP (crossed circles) or placebo (PLC; black circles) from day 1 until day 8 postinfection (p.i.). At day 9 p.i., secretion of the pro-inflammatory cytokine IFN- $\gamma$ was determined in ex vivo lung biopsies. Naive mice (i.e., without ileitis and without treatment; white circles) served as negative controls. Numbers of animals (in parentheses), means, and significance levels ( $p$ values) determined by Mann-Whitney $U$ test are indicated. Data shown were pooled from four independent experiments

coli; $p<0.05-0.01$; Figure 7B) and of enterococci in their ileal lumen $(p<0.005-0.001$; Figure 7C), whereas small intestinal numbers of (potentially probiotic) bifidobacteria $(p<0.05-0.001$; Figure 7E) and of Clostridium coccoides group members $(p<$ 0.05-0.001; Figure 7G) were lower as compared to naive controls. Notably, decreases in ileal bifidobacterial counts were less pronounced upon NAP as compared to placebo treatment $(p<0.05$; Figure 7E). Furthermore, Clostridium leptum loads were slightly decreased in the small intestines of placebo, but not NAPtreated hma mice at day 9 p.i. $(p<0.005$ vs. naive; Figure $7 \mathrm{H})$.

Thus, anti-inflammatory effects exerted by NAP were accompanied by less distinct inflammation-induced decreases in ileal bifidobacteria during subacute ileitis in hma mice.

\section{Discussion}

Many in vitro and in vivo studies have shown that the octapeptide NAP exerts its neuroprotective effects via immunomodulatory and anti-oxidative mechanisms [10]. Very recently, our group showed for the first time that the beneficial actions of NAP were not restricted to the nervous system but could also be observed during inflammatory conditions of the intestinal tract $[11,12]$. Given that either applied murine inflammation model (i.e., acute ileitis following peroral high-dose $T$. gondii infection and acute dextran sulfate sodium [DSS] induced colitis) was lethal within 1 week, the here presented results provide additional insights into the anti-inflammatory properties of NAP in a far less acute gut inflammation model. The applied subacute ileitis model following peroral low-dose $T$. gondii infection of (with respect to their gut microbiota) "humanized" mice 
A IL-10 - COLON

$\mathrm{p}<0.005$

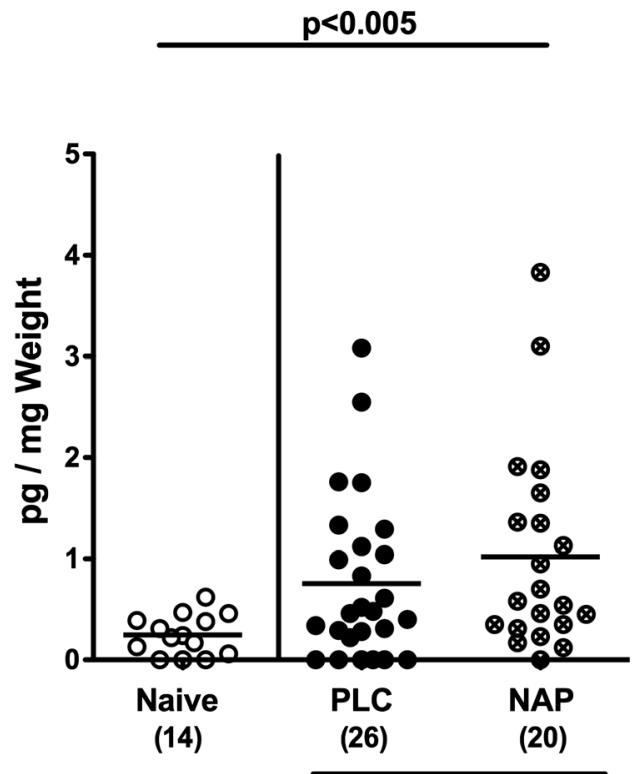

T. gondii
B

IL-10 - SERUM

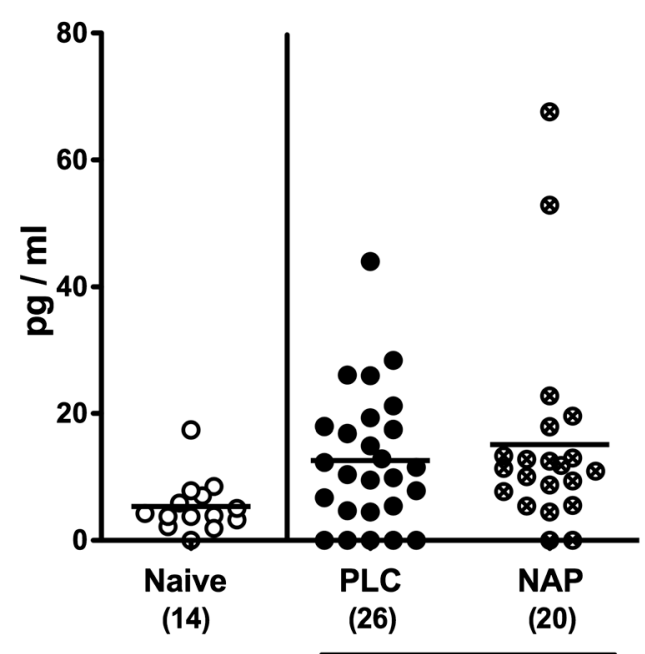

T. gondii

Figure 6. Intestinal and systemic anti-inflammatory cytokine responses upon NAP treatment of human microbiota-associated mice suffering from subacute ileitis. Subacute ileitis was induced in human microbiota-associated (hma) mice on day 0 by peroral low-dose $T$. gondii infection as described in Materials and Methods section. Hma mice were then treated with synthetic NAP (crossed circles) or placebo (PLC; black circles) from day 1 until day 8 postinfection (p.i.). At day 9 p.i., secretion of the anti-inflammatory cytokine IL-10 was determined in (A) ex vivo colon biopsies and (B) serum samples. Naive mice (i.e., without ileitis and without treatment; white circles) served as negative controls. Numbers of animals (in parentheses), means, and significance levels ( $p$ values) determined by Mann-Whitney $U$ test are indicated. Data shown were pooled from four independent experiments

model has been very recently introduced to the scientific community [13] (within this issue) and provides valuable opportunities to elucidate the molecular mechanisms underlying the interplay between pathogen(s), the human commensal gut microbiota, and host immune responses during intestinal inflammation.

In the present study, we show that NAP exerts anti-inflammatory properties during subacute ileitis of "humanized" mice as indicated by (1) lower intestinal numbers of T and B lymphocytes; (2) less secretion of pro-inflammatory IFN- $\gamma$ by mesenteric lymph nodes; (3) lower pro-inflammatory cytokine levels in extra-intestinal compartments including liver, kidney, and lung; (4) augmented colonic as well as systemic secretion of the anti-inflammatory cytokine IL-10; and (5) higher (probiotic) bifidobacterial loads in the ileal lumen following NAP as compared to placebo treatment.

The better outcome in NAP-treated mice was due to an alleviated Th1-type immune response as indicated by less pronounced small and large intestinal accumulation of $\mathrm{T}$ lymphocytes, the major driving forces of $T$. gondii-induced ileitis [28] and lower intestinal IFN- $\gamma$ concentrations, which is in line with results obtained from our previous acute inflammation models with lethal outcome $[11,12]$. In support, NAP was shown to suppress proliferation and activation of $\mathrm{T}$ cells in vitro leading to a dampened secretion of pro-inflammatory mediators including IFN- $\gamma$ [10]. Remarkably, the anti-inflammatory properties of NAP were not restricted to the intestinal tract but were also effective in extra-intestinal including systemic compartments. Lower concentrations of pro-inflammatory cytokines in liver and kidney of NAP as compared to placebo-treated mice suffering from intestinal inflammation are supported by our previous studies $[11,12]$. The anti-inflammatory effects of NAP were not only due to less distinct expression of pro-inflammatory mediators but also to increased intestinal as well as systemic secretion of the anti-inflammatory cytokine IL-10. Particularly, the pronounced systemic anti-inflammatory response observed in serum samples taken from NAP-treated mice in our present report is remarkable. ADNP is hypothesized to be a secreted protein $[2,29]$, and NAP, to exert its immunoregulatory properties in the circulation in a cytokine-like (apocrine or paracrine) manner, but without a defined surface receptor [3].

One might assume that the better outcome of mice following NAP treatment might be due to less induced small intestinal inflammation following T. gondii infection. Notably, we assured that NAP application did not interfere with $T$. gondii infection and hence the induction of small intestinal inflammation. First, to avoid direct NAP-parasite-interaction, synthetic NAP treatment was initiated $24 \mathrm{~h}$ after peroral T. gondii challenge; and second, comparable parasitic DNA loads were measured in the ilea of NAP and placebo-treated mice in our previous [11] and actual study (not shown).

Furthermore, we ruled out that NAP exerts any anti-bacterial effects in vitro, given that the distinct gut microbiota composition plays a critical role in initiation, perpetuation, and limitation of immunopathological conditions [11, 26, 27, 30-34]. Interestingly, subacute ileitis development was accompanied by profound shifts in gut microbiota composition that were characterized by higher loads of potentially pro-inflammatory enterobacteria such as $E$. coli and enterococci within the inflamed ileal lumen, whereas anaerobic bacterial groups such as $C$. coccoides and bifidobacteria were far less abundant as compared to naive mice. These results are supported by the small intestinal commensal bacterial changes observed in hyper-acute ileitis following high-dose T. gondii infection of both conventionally colonized and human microbiota-associated mice [17, 23, 33-37].

Remarkably, bifidibacterial loads were higher in the small intestines of NAP as compared to placebo-treated mice with 
A Total Eubacterial Load (ILEUM)

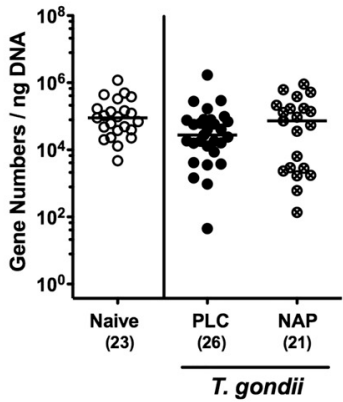

E

E Bifidobacteria

(ILEUM)

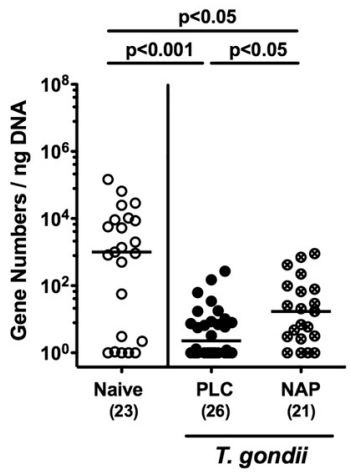

B

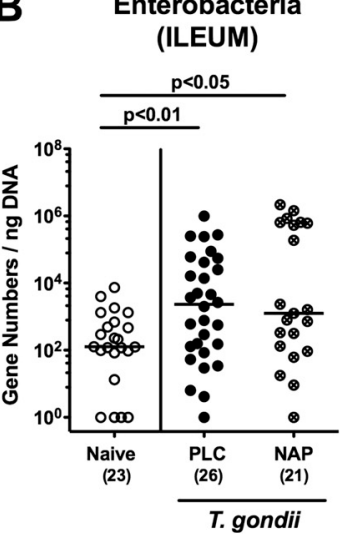

F Bacteroides/Prevotella (ILEUM)

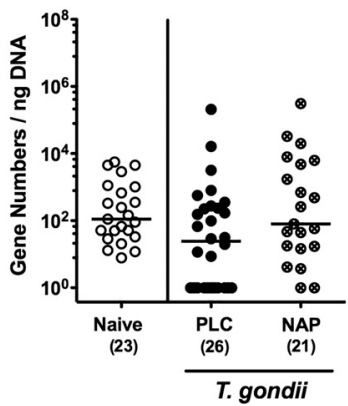

C

Enterococci

(ILEUM)

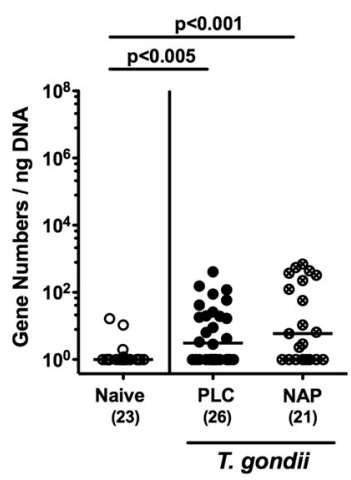

G Clostridium coccoides (ILEUM)

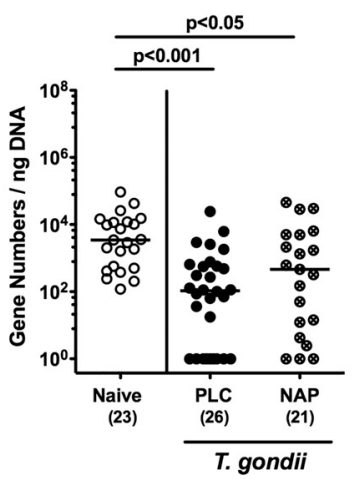

D

Lactobacilli

(ILEUM)

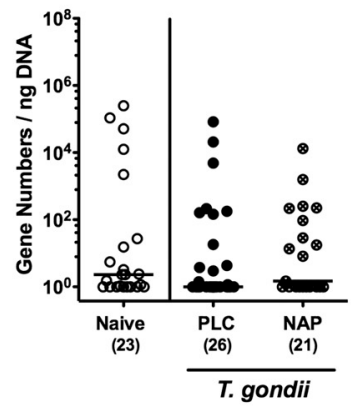

H

Clostridium leptum (ILEUM)

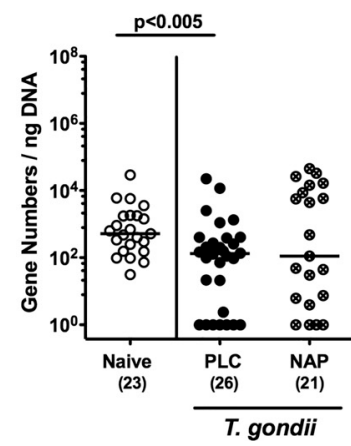

Figure 7. Ileal microbiota composition in NAP-treated mice with a human gut microbiota suffering from subacute ileitis. Subacute ileitis was induced in human microbiota-associated (hma) mice on day 0 by peroral low-dose $T$. gondii infection as described in Materials and Methods section. Hma mice were then treated with synthetic NAP (crossed circles) or placebo (PLC; black circles) from day 1 until day 8 postinfection (p.i.). The main gut bacterial groups within the ileum lumen of hma mice were quantitatively assessed applying culture-independent (i.e., molecular $16 \mathrm{~S}$ rRNA based) methods at day 9 p.i. Naive mice (without ileitis and without treatment; white circles) served as negative controls. 16S rRNA of the total eubacterial loads as well as of the main intestinal bacterial groups including enterobacteria, enterococci, lactobacilli, bifidobacteria, Bacteroides/Prevotella species, Clostridium coccoides group and Clostridium leptum group are expressed as gene numbers per nanogram DNA. Medians (black bars) and significance levels ( $p$ values) determined by Mann-Whitney $U$ test are indicated. Data shown are pooled from three independent experiments

subacute ileitis. This observation is well in line with results obtained from our recent study where NAP-treated mice were not only less suffering from acute colitis but also displayed higher fecal bifidobacterial loads [12]. Bifidobacteria belong to the phylum Actinobacteria and constitute anaerobic Grampositive commensal bacteria fulfilling a multitude of metabolic functions such as production of fatty acids and vitamins, degradation of oxalate, and hydrolysis of bile salts, for instance [38]. Furthermore, bifidobacteria are known to exhibit probiotic (i.e., health-promoting) and particularly anti-inflammatory properties [38]. Until now, we cannot decipher, however, whether the higher bifidobacetrial numbers in NAP-treated mice were due to a direct NAP effect or a bystander-effect during a less severe intestinal inflammatory process following NAP treatment, or both ("hen-and-egg-paradigm"). Nevertheless, very recently, a distinct Bifidobacterium dentium strain was shown to modulate visceral sensitivity in the intestines by production of the primary inhibitory neurotransmitter within the mammalian CNS, namely, $\gamma$-aminobutyric acid (GABA), thereby providing a direct link between bifidobacteria and neuromodulatory activities [39]. This study (in line with our previous [12] and present report) further emphasizes the (patho-)physiological importance of the gut-brain axis.

It is tempting to speculate whether the observed anti-inflammatory effects of NAP might have been even more pronounced upon increases in dosage and/or application frequencies of the synthetic compound or upon a prophylactic application regimen starting before induction of disease.
In summary, the octapeptide NAP exerts intestinal as well as extra-intestinal including systemic anti-inflammatory effects in "humanized" mice suffering from subacute ileitis. Beneficial effects of NAP treatment/prophylaxis further need to be addressed in chronic gut inflammation models.

We conclude that our findings further support that NAP might be regarded as future treatment option directed against intestinal inflammation.

\section{Funding Sources}

This work was supported by grants from the German Research Foundation (DFG) to S.B. (SFB633, TP A7), M.M.H. (SFB633, TP B6 and SFB TR84, TP A5), and U.F. (SFB633, TP B6) and from the German Federal Ministry of Education and Research (BMBF) to S.B. (TP1.1). The funders had no role in study design, data collection and analysis, decision to publish, or preparation of the article.

\section{Authors' Contributions}

Conceived and designed the experiments: SB IG MMH

Performed the experiments: UE MMH

Analyzed the data: $\mathrm{MMH}$

Critically discussed the data: EG IRD SB IG MMH

Wrote the paper: MMH

Co-edited the paper: SB IG 


\section{Conflicts of Interest}

M.M.H., I.R.D., and S.B. are editorial board members.

Acknowledgments. The authors thank Alexandra BittroffLeben, Ines Puschendorf, Gernot Reifenberger, and the staff of the animal research facility of the Charite - University Medicine Berlin for excellent technical assistance and animal breeding.

\section{References}

1. Bassan M, Zamostiano R, Davidson A, Pinhasov A, Giladi E, Perl O, et al. Complete sequence of a novel protein containing a femtomolar-activitydependent neuroprotective peptide. J Neurochem. 1999;8:1283-93.

2. Zamostiano R, Pinhasov A, Gelber E, Steingart RA, Seroussi E, Giladi E, et al. Cloning and characterization of the human activity-dependent neuroprotective protein. J Biol Chem. 2001;276:708-14.

3. Gozes I, Divinski I. The femtomolar-acting NAP interacts with microtubules: novel aspects of astrocyte protection. J Alzheimers Dis. 2004;6:S37-41.

4. Quintana FJ, Zaltzman R, Fernandez-Montesinos R, Herrera JL, Gozes I, Cohen IR, et al. NAP, a peptide derived from the activity-dependent neuroprotective protein, modulates macrophage function. Ann N Y Acad Sci. 2006;1070:500-6.

5. Beni-Adani L, Gozes I, Cohen Y, Assaf Y, Steingart RA, Brenneman DE, et al. A peptide derived from activity-dependent neuroprotective protein (ADNP) ameliorates injury response in closed head injury in mice. J Pharmacol Exp Ther. 2001;296:57-63.

6. Zemlyak I, Furman S, Brenneman DE, Gozes I. A novel peptide prevents death in enriched neuronal cultures. Regul Pept. 2000;96:39-43.

7. Ashur-Fabian O, Segal-Ruder Y, Skutelsky E, Brenneman DE, Steingart RA, Giladi E, et al. The neuroprotective peptide NAP inhibits the aggregation of the beta-amyloid peptide. Peptides. 2003;24:1413-23.

8. Rotstein M, Bassan H, Kariv N, Speiser Z, Harel S, Gozes I. NAP enhances neurodevelopment of newborn apolipoprotein E-deficient mice subjected to hypoxia. J Pharmacol Exp Ther. 2006;319:332-9.

9. Greggio S, de Paula S, de Oliveira IM, Trindade C, Rosa RM, Henriques $\mathrm{JA}$, et al. NAP prevents acute cerebral oxidative stress and protects against longterm brain injury and cognitive impairment in a model of neonatal hypoxiaischemia. Neurobiol Dis. 2011;44:152-9.

10. Braitch M, Kawabe K, Nyirenda M, Gilles LJ, Robins RA, Gran B, et al. Expression of activity-dependent neuroprotective protein in the immune system: possible functions and relevance to multiple sclerosis. Neuroimmunomodulation. 2010;17:120-5

11. Heimesaat MM, Fischer A, Kuhl AA, Gobel UB, Gozes I, Bereswill S. Anti-inflammatory properties of NAP in acute Toxoplasma gondii-induced ileitis in mice. Eur J Microbiol Immunol. 2015:5:210-20.

12. Heimesaat MM, Giladi E, Kuhl AA, Bereswill S, Gozes I. The octapeptide NAP alleviates intestinal and extra-intestinal anti-inflammatory sequelae of acute experimental colitis. Peptides. 2018;101:1-9.

13. Heimesaat MM, Escher U, Grunau A, Fiebiger U, Bereswill S. Peroral low-dose Toxoplasma gondii infection of human microbiota asociated mice a subacute ileitis model to unravel pathogen-host interaction. Eur J Microbiol Immunol. 2018. EUJMI-D-18-00005.

14. Bereswill S, Fischer A, Plickert R, Haag LM, Otto B, Kuhl AA, et al. Novel murine infection models provide deep insights into the "menage a trois" of Campylobacter jejuni, microbiota and host innate immunity. PLoS One. 2011;6:e20953.

15. von Klitzing E, Ekmekciu I, Bereswill S, Heimesaat MM. Acute ileitis facilitates infection with multidrug resistant Pseudomonas aeruginosa in human microbiota-associated mice. Gut Pathog. 2017;9:4

16. von Klitzing E, Oz F, Ekmekciu I, Escher U, Bereswill S, Heimesaat MM. Comprehensive survey of intestinal microbiota changes in offspring of human microbiota-associated mice. Eur J Microbiol Immunol. 2017;7:65-75.

17. Heimesaat MM, Bereswill S, Fischer A, Fuchs D, Struck D, Niebergall J, et al. Gram-negative bacteria aggravate murine small intestinal Th1-type immunopathology following oral infection with Toxoplasma gondii. J Immunol. 2006;177:8785-95.

18. Heimesaat MM, Fischer A, Jahn HK, Niebergall J, Freudenberg M, Blaut M, et al. Exacerbation of murine ileitis by Toll-like receptor 4 mediated sensing of lipopolysaccharide from commensal Escherichia coli. Gut. 2007;56:941-8.

19. Heimesaat MM, Nogai A, Bereswill S, Plickert R, Fischer A, Loddenkemper C, et al. MyD88/TLR9 mediated immunopathology and gut microbiota dynamics in a novel murine model of intestinal graft-versus-host disease. Gut. 2010;59:1079-87.

20. Alutis ME, Grundmann U, Fischer A, Kuhl AA, Bereswill S, Heimesaat MM. Selective gelatinase inhibition reduces apoptosis and pro-inflammatory immune cell responses in Campylobacter jejuni-infected gnotobiotic IL-10 deficient mice. Eur J Microbiol Immunol. 2014;4:213-22.

21. Munoz M, Heimesaat MM, Danker K, Struck D, Lohmann U, Plickert R, et al. Interleukin (IL)-23 mediates Toxoplasma gondii-induced immunopathology in the gut via matrixmetalloproteinase-2 and IL-22 but independent of IL-17. J Exp Med. 2009;206:3047-59.

22. Heimesaat MM, Fischer A, Siegmund B, Kupz A, Niebergall J, Fuchs D, et al. Shift towards pro-inflammatory intestinal bacteria aggravates acute murine colitis via Toll-like receptors 2 and 4. PLoS One. 2007;2:e662.

23. Bereswill S, Kuhl AA, Alutis M, Fischer A, Mohle L, Struck D, et al. The impact of Toll-like-receptor-9 on intestinal microbiota composition and extra-intestinal sequelae in experimental Toxoplasma gondii induced ileitis. Gut Pathog. 2014;6:19.

24. Heimesaat MM, Boelke S, Fischer A, Haag LM, Loddenkemper C, Kuhl AA, et al. Comprehensive postmortem analyses of intestinal microbiota changes and bacterial translocation in human flora associated mice. PLoS One. 2012; 7:e40758.

25. Rausch S, Held J, Fischer A, Heimesaat MM, Kuhl AA, Bereswill S, et al. Small intestinal nematode infection of mice is associated with increased enterobacterial loads alongside the intestinal tract. PLoS One. 2013;8:e74026.

26. Thoene-Reineke C, Fischer A, Friese C, Briesemeister D, Gobel UB, Kammertoens $\mathrm{T}$, et al. Composition of intestinal microbiota in immune-deficient mice kept in three different housing conditions. PLoS One. 2014;9:e113406.

27. Heimesaat MM, Reifenberger G, Vicena V, Illes A, Horvath G, Tamas A, et al. Intestinal microbiota changes in mice lacking pituitary adenylate cyclase activating polypeptide (PACAP) - bifidobacteria make the difference. Eur J Microbiol Immunol. 2017:7:187-99.

28. Munoz M, Liesenfeld O, Heimesaat MM. Immunology of Toxoplasma gondii. Immunol Rev. 2011;240:269-85.

29. Furman S, Steingart RA, Mandel S, Hauser JM, Brenneman DE, Gozes I. Subcellular localization and secretion of activity-dependent neuroprotective protein in astrocytes. Neuron Glia Biol. 2004;1:193-9.

30. Sartor RB. Microbial influences in inflammatory bowel diseases. Gastroenterol. 2008;134:577-94.

31. Sartor RB. Genetics and environmental interactions shape the intestinal microbiome to promote inflammatory bowel disease versus mucosal homeostasis. Gastroenterol. 2010;139:1816-9.

32. Heimesaat MM, Fischer A, Alutis M, Grundmann U, Boehm M, Tegtmeyer N, et al. The impact of serine protease HtrA in apoptosis, intestinal immune responses and extra-intestinal histopathology during Campylobacter jejuni infection of infant mice. Gut Pathog. 2014;6:16.

33. Heimesaat MM, Dunay IR, Alutis M, Fischer A, Mohle L, Gobel UB, et al. Nucleotide-oligomerization-domain-2 affects commensal gut microbiota composition and intracerebral immunopathology in acute Toxoplasma gondii induced murine ileitis. PLoS One. 2014;9:e105120.

34. Fiebiger U, Bereswill S, Heimesaat MM. Dissecting the interplay between intestinal microbiota and host immunity in health and disease: lessons learned from germfree and gnotobiotic animal models. Eur J Microbiol Immunol. 2016;6:253-71.

35. Erridge C, Duncan SH, Bereswill S, Heimesaat MM. The induction of colitis and ileitis in mice is associated with marked increases in intestinal concentrations of stimulants of TLRs 2, 4, and 5. PLoS One. 2010;5:e9125.

36. Heimesaat MM, Dunay IR, Schulze S, Fischer A, Grundmann U, Alutis M, et al. Pituitary adenylate cyclase-activating polypeptide ameliorates experimental acute ileitis and extra-intestinal sequelae. PLoS One. 2014;9:e108389.

37. von Klitzing E, Ekmekciu I, Kuhl AA, Bereswill S, Heimesaat MM. Intestinal, extra-intestinal and systemic sequelae of Toxoplasma gondii induced acute ileitis in mice harboring a human gut microbiota. PLoS One. 2017;12: e0176144.

38. Klijn A, Mercenier A, Arigoni F. Lessons from the genomes of bifidobacteria. FEMS Microbiol Rev. 2005;29:491-509.

39. Pokusaeva K, Johnson C, Luk B, Uribe G, Fu Y, Oezguen N, et al. GABA-producing Bifidobacterium dentium modulates visceral sensitivity in the intestine. Neurogastroenterol Motil. 2017;29. 